\title{
THE EFFECT OF RECEIVER DIVERSITY COMBINING ON OPTIMUM ENERGY ALLOCATION AND ENERGY EFFICIENCY OF COOPERATIVE WIRELESS TRANSMISSION SYSTEMS
}

\author{
Jie Yang and D. Richard Brown III \\ $\{$ abbyyang,drb\}@wpi.edu \\ Electrical and Computer Engineering Department, Worcester Polytechnic Institute, Worcester, MA.
}

\begin{abstract}
This paper considers the problem of how to efficiently allocate transmission energy in a cooperative wireless communication system with one source, one relay, and one destination. The relay assists the source via an orthogonal amplify-and-forward protocol. The channels are assumed to be flat fading and the destination combines the source and relay transmissions using either maximal ratio combining (MRC) or equal gain combining (EGC).

We analyze the problem of minimizing average total (source plus relay) transmit energy under a fixed outage probability constraint. We show that the choice of receiver diversity combining technique affects both the optimum energy allocation and the overall energy efficiency of the cooperative communication system. Our results show that the ratio of optimum relay to source transmission energy tends to be greater for EGC than MRC. Our results also show that, while cooperative transmission with MRC is always the most efficient strategy, cooperative transmission with EGC tends to be more energy efficient than direct transmission when the outage probability constraint is small or when the relay experiences an advantaged channel to the destination.
\end{abstract}

Index Terms - Cooperative systems, resource allocation

\section{INTRODUCTION}

Spatial diversity can be achieved in multiuser communication systems through user cooperation where transmitters and/or receivers pool their antenna resources to form a "virtual antenna array". Transmit cooperation was first proposed in [1] and has since been shown to offer many of the benefits of multi-antenna transmission, e.g. increased rate and/or reduced outage probability, to transmitters with single antennas [2]-[4].

Unlike a single transmitter employing a conventional antenna array, cooperating transmitters each have their own local resources. It was first suggested in [1] that cooperation could lead to an overall reduction in transmit energy and, consequently, increased battery life for wireless transmitters. Recent results have shown this prediction to be true even for simple cooperative protocols such as amplify-and-forward [5]-[7] and decode-and-forward [8]-[10]. These results also demonstrated the somewhat surprising result that, while knowledge of channel state information at the transmitters (CSIT) is beneficial, it is not necessary to achieve significant gains in energy efficiency with respect to direct (non-cooperative) transmission.

While recent work in this area has focused on the impact of the cooperative protocol and CSIT, the impact of receiver diversity combining on optimum energy allocation and overall energy

This work was supported by NSF award CCF-0447743. efficiency has not been investigated. In this paper, we analyze how the receiver diversity combining technique affects both the optimum energy allocation and the overall energy efficiency of amplify-and-forward cooperative transmission systems. We focus on a comparison of maximal ratio combining (MRC) and equal gain combining (EGC) for a system with flat fading channels and a fixed outage probability constraint. We derive the optimum energy allocation strategy for EGC and compare the energy efficiency and allocation to the MRC results derived in [7]. Our results show that, unlike MRC, optimum cooperative transmission with EGC always requires transmission by the relaying node. Numerical results for independent Rayleigh fading channels show that the ratio of optimum relay to source transmission energy tends to be greater for EGC than MRC. We also show that, while cooperative transmission with MRC is always the most efficient strategy, cooperative transmission with EGC tends to be more energy efficient than direct transmission when the outage probability constraint is small or when the relaydestination channel is advantaged.

\section{SYSTEM MODEL AND COOPERATIVE PROTOCOL}

We consider the cooperative transmission system shown in Figure 1 where a source $(S)$ communicates to a destination $(D)$ with potential assistance from a single relay $(R)$. All of the channels in Figure 1 are assumed to be frequency non-selective and the channel magnitudes $\left|g_{s}\right|,\left|g_{r}\right|$, and $|h|$ are assumed to be independent Rayleigh distributed random variables. The channels are also assumed to be quasi-static in the sense that the channel realizations remain constant over the duration of a cooperative transmission interval but are i.i.d. for different transmission intervals.

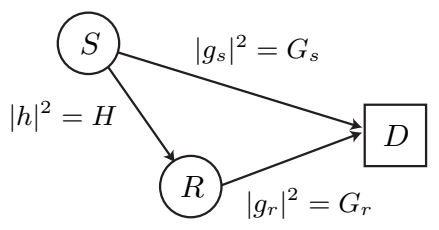

Fig. 1. Cooperative transmission system model.

The source is assumed to have a strict transmission delay constraint in the sense that it must transmit a symbol or codeword in each transmission interval. We assume that the relay cooperates with the source through the half-duplex "amplify-andforward" (AF) protocol first described in [11]. In the AF protocol, the transmission interval is divided into two time-slots of equal duration. In the first timeslot, the source transmits the symbol (or codeword) $\boldsymbol{x}$. This transmission is received by the destination and 
the relay through channels $g_{s}$ and $h$, respectively. We assume that the receivers know the appropriate channel phases and correct the received phase prior to subsequent processing or retransmission. The destination and relay observations in the first timeslot can be written in standard signal space notation as

$$
\begin{aligned}
& \boldsymbol{y}_{d 1}=\left|g_{s}\right| a_{s} \boldsymbol{x}+\boldsymbol{w}_{d 1} \text { (destination) } \\
& \boldsymbol{y}_{r 1}=|h| a_{s} \boldsymbol{x}+\boldsymbol{w}_{r 1} \text { (relay) }
\end{aligned}
$$

where $a_{s}$ is the amplitude of the source's transmission and $\boldsymbol{w}_{d 1}$ and $\boldsymbol{w}_{r 1}$ are zero-mean unit-variance independent complex Gaussian random vectors resulting from additive white Gaussian noise at the input of the destination and relay receivers, respectively.

In the second timeslot, the relay retransmits the phase corrected signal that it observed in the first timeslot and this signal is received by the destination through channel $g_{r}$. The destination's observation in the second timeslot can be written as

$\boldsymbol{y}_{d 2}=\left|g_{r}\right| a_{r} \boldsymbol{y}_{r 1}+\boldsymbol{w}_{d 2}=\left|g_{r}\right| a_{r}|h| a_{s} \boldsymbol{x}+\left|g_{r}\right| a_{r} \boldsymbol{w}_{r 1}+\boldsymbol{w}_{d 2}$

where $a_{r}$ is the amplitude of the relay's transmission and $\boldsymbol{w}_{d 2}$ denotes the receiver noise at the destination in the second timeslot.

\section{SNR ANALYSIS}

The SNR analysis in this section follows the approach developed in [7]. To isolate the effect of the receiver diversity combining technique, we assume that the source and the relay have access to the channel amplitudes and are able to dynamically allocate their transmission energies according to the instantaneous channel amplitudes ${ }^{1}$ in each transmission interval.

When the destination has full access to the channel state information (CSI) and transmit energies, MRC can be applied to the relevant source/relay observations in both timeslots to maximize the SNR. The resulting instantaneous SNR at the destination, after $\mathrm{MRC}$, can be expressed as [7]

$$
\mathrm{SNR}_{\mathrm{mrc}}=G_{s} \mathcal{E}_{s}+\frac{H \mathcal{E}_{s} G_{r} \mathcal{E}_{r}}{1+H \mathcal{E}_{s}+G_{r} \mathcal{E}_{r}}
$$

where $\mathcal{E}_{s}=a_{s}^{2} \mathrm{E}\left[\boldsymbol{x}^{H} \boldsymbol{x}\right]$ is the source transmission energy and $\mathcal{E}_{r}=$ $a_{r}^{2}\left(H \mathcal{E}_{s}+1\right)$ is the relay transmission energy. Notice that the first part of (1) is the SNR of direct transmission.

When CSI is not available at the destination, MRC cannot be used. One approach in this scenario is to combine the observations with equal gain, i.e. EGC. The resulting instantaneous SNR at the destination, after EGC, can be expressed as

$\mathrm{SNR}_{\mathrm{egc}}=\frac{G_{s} \mathcal{E}_{s}}{2}+\frac{\mathcal{E}_{s} G_{r} \mathcal{E}_{r}\left(H-\frac{G_{s}}{2}\right)+2 \mathcal{E}_{s}\left(G_{r} G_{s} \mathcal{E}_{r} H \psi\right)^{1 / 2}}{2 \psi+G_{r} \mathcal{E}_{r}}$,

where $\psi=H \mathcal{E}_{s}+1$. The following sections analyze optimum energy allocation strategies and energy efficiency of the two-source cooperative transmission system based on (1) and (2).

\section{OPTIMUM ENERGY ALLOCATION STRATEGIES}

The performance measure that we consider is outage probability, defined as the probability that the SNR at the destination falls below a threshold $\rho$, i.e. $p:=\operatorname{Prob}[$ outage $]=\operatorname{Prob}[\mathrm{SNR}<\rho]$. The following sections analyze the problem of minimizing average total transmit energy under a fixed outage probability constraint for a destination using EGC or MRC.

\footnotetext{
${ }^{1}$ These channel amplitudes could, for instance, be provided through separate low-rate feedback channels.
}

\section{IV-A. Equal Gain Combining}

Using the approach in [7], we first consider the case $p=0$. In this case, minimizing the instantaneous total energy is equivalent to minimizing the average total energy. The relay node energy $\mathcal{E}_{r}$ can be written as a function of $\rho$ and $\mathcal{E}_{s}$ by solving (2) for $\mathcal{E}_{r}$ when $\mathrm{SNR}_{\text {egc }}=\rho$. The solution yields two roots for $\mathcal{E}_{r}$. The correct root yields $\mathcal{E}_{r}=0$ when $\mathcal{E}_{s}=\frac{2 \rho}{G_{s}}$ and can be written as

$$
\begin{aligned}
\mathcal{E}_{\mathrm{r}}= & \frac{\left(H \mathcal{E}_{s}+1\right)\left(G_{s} H \mathcal{E}_{s}^{2}+2 \rho H \mathcal{E}_{s}+G_{s} \mathcal{E}_{s} \rho-2 \rho^{2}\right)}{G_{r}\left(\rho-H \mathcal{E}_{s}\right)^{2}} \\
& -\frac{2 \mathcal{E}_{s}\left(H \mathcal{E}_{s}+1\right)\left[G_{s} H \rho\left(2 H \mathcal{E}_{s}+G_{s} \mathcal{E}_{s}-2 \rho\right)\right]^{1 / 2}}{G_{r}\left(\rho-H \mathcal{E}_{s}\right)^{2}}
\end{aligned} .
$$

The admissible range of instantaneous energy allocations that satisfy $\mathrm{SNR}_{\text {egc }}=\rho$ can be described as the region in $\mathbb{R}^{2}$ where $\mathcal{E}_{r} \geq 0$ and $\frac{2 \rho}{2 H+G_{s}} \leq \mathcal{E}_{s} \leq \frac{2 \rho}{G_{s}}$. The case $\mathcal{E}_{r}=0$ establishes the upper limit on the interval of admissible solutions for $\mathcal{E}_{s}$. The lower limit on the interval is established by the requirement for total energy to be a real-valued quantity. The square root in the numerator of (3) reveals that $\mathcal{E}_{r} \in \mathbb{R}$ only if $\mathcal{E}_{s} \geq \frac{2 \rho}{2 H+G_{s}}$.

Denote the admissible range of $\mathcal{E}_{s}$ as $\mathcal{A}$. Given $\rho$ and the squared channel amplitudes $G_{s}, G_{r}$, and $H$, (3) implies that $\mathcal{E}_{r}$ is dependent on $\mathcal{E}_{s}$. Hence, the total energy $\mathcal{E}_{\text {egc }}$ is dependent on $\mathcal{E}_{s}$ and the instantaneous total energy minimization problem can be stated as

$$
\mathcal{E}_{\text {egc }}^{*}=\min _{\mathcal{E}_{s} \in \mathcal{A}} \mathcal{E}_{\text {egc }} .
$$

It can be shown that an explicit analytical solution to (4) requires computing the roots of a quartic polynomial. Numerical solutions to (4), however, are aided by the following result ${ }^{2}$.

Proposition 1: Total energy $\mathcal{E}_{\text {egc }}$ is a convex function of $\mathcal{E}_{s}$ on $\mathcal{A}$.

Proposition 1 implies that standard numerical convex optimization methods can be used to find the unique solution to (4).

Denote $\mathcal{E}_{s}^{*}$ as the value of $\mathcal{E}_{s}$ that attains the minimum in (4) and note that $\mathcal{E}_{r}^{*}$ is implied by (3). The following proposition shows that the relay should always transmit, i.e. $\mathcal{E}_{r}^{*}>0$ for all channel states, when the destination uses EGC. This is in contrast to the result in [7] showing that direct transmission $\left(\mathcal{E}_{r}^{*}=0\right)$ is optimum for certain channel states when the destination uses MRC.

Proposition 2: $\mathcal{E}_{r}^{*}>0$ for all values of $G_{s}, G_{r}, H, \rho$ when the destination uses EGC combining.

We now consider the optimum energy allocation strategy for the case when $p>0$. Note that the random nature of the channel state implies that $\mathcal{E}_{\text {egc }}^{*}$ is random. We denote the cdf of $\mathcal{E}_{\text {egc }}^{*}$ satisfying $\mathrm{SNR}_{\text {egc }}=\rho$ as $F_{\mathcal{E}_{\text {egc }}^{*}}(x):=\operatorname{Prob}\left[\mathcal{E}_{\text {egc }}^{*} \leq x\right]$. Let $t$ denote the value at which $F_{\mathcal{E}_{\text {egc }}^{*}}(t)=1-p$. Given the current channel state, solve for the optimum transmission energies $\mathcal{E}_{s}^{*}$ and $\mathcal{E}_{r}^{*}$ that satisfy $\mathrm{SNR}_{\text {egc }}=\rho$ via (2), (3), and (4). Note that the resulting minimum instantaneous total energy $\mathcal{E}_{\text {egc }}^{*}$ will exceed the threshold $t$ with probability $p$. Since outage events are permitted with probability $p$, the strategy that minimizes average total transmission energy is to not transmit if $\mathcal{E}_{\text {egc }}^{*}>t$. If $\mathcal{E}_{\text {egc }}^{*} \leq t$, transmission occurs such that $\mathrm{SNR}_{\text {egc }}=\rho$ with the optimum energies $\mathcal{E}_{s}^{*}$ and $\mathcal{E}_{r}^{*}$. We note that this is an opportunistic transmission strategy where the source and relay avoid transmission (and cause an outage) in cases when the channel state is unfavorable. The outage probability requirement is satisfied under this strategy since the SNR at the destination will be equal to $\rho$ with probability $1-p$ and equal to zero otherwise.

\footnotetext{
${ }^{2}$ Proofs of Propositions I and II are given in the Appendices.
} 


\section{IV-B. Maximal Ratio Combining}

Optimum energy allocation for the two-source, one-destination scenario with MRC was analyzed in [7]. This section summarizes the main results to facilitate a comparison with EGC in Section V.

For the case when $p=0$, it was shown in [7] that the source should use direct transmission, i.e. $\mathcal{E}_{r}^{*}=0$ and $\mathcal{E}_{s}^{*}=\frac{\rho}{G_{s}}$, when the channel state satisfies $\frac{G_{r}}{G_{s}} \leq 1+\frac{G_{s}}{H \rho}$. When the channel state does not satisfy this condition, cooperative transmission is more energy efficient than direct transmission. The source energy $\mathcal{E}_{s}^{*}$ that minimizes $\mathcal{E}_{\mathrm{mrc}}$ while satisfying $\mathrm{SNR}_{\mathrm{mrc}}=\rho$ is

$$
\mathcal{E}_{s}^{*}=\frac{\rho}{H+G_{s}}+\frac{(\rho H)^{1 / 2}\left(G_{s}+(1+\rho) H\right)^{1 / 2}}{\left(H+G_{s}\right)\left(H\left(G_{r}-G_{s}\right)+G_{s} G_{r}\right)^{1 / 2}} .
$$

The optimum relay energy $\mathcal{E}_{r}^{*}$ is implied through (1) and (5) given $\mathrm{SNR}_{\text {mrc }}=\rho$. The minimum total transmission energy is then $\mathcal{E}_{\text {mrc }}^{*}=\mathcal{E}_{s}^{*}+\mathcal{E}_{r}^{*}$.

The optimum energy allocation strategy for the case $p>0$ follows the EGC case, replacing $\mathcal{E}_{\text {egc }}^{*}$ with $\mathcal{E}_{\text {mrc }}^{*}$, and noting that the relay does not transmit when $\frac{G_{r}}{G_{s}}<1+\frac{G_{s}}{H \rho}$.

\section{NUMERICAL EXAMPLES}

This section presents numerical examples demonstrating the impact of receiver diversity combining on optimum cooperative energy allocation and energy efficiency for the case when the channels are Rayleigh fading and independent. Denote $\mu_{r}, \mu_{s}$, and $\mu_{H}$ as the mean of the exponential random variables $G_{r}, G_{s}$, and $H$. All of the results in this section assume $\mu_{H}=100$, and $\rho=10 \mathrm{~dB}$. Figures 2 and 3 consider three separate cases: (i) when the relay has a statistically advantaged channel to the destination, i.e. $\mu_{r}=100$ and $\mu_{s}=10$; (ii) when the source and relay face statistically symmetric Rayleigh fading channels to the destination, i.e. $\mu_{r}=\mu_{s}=10$; and (iii) when the relay has a statistically disadvantaged channel to the destination, i.e. $\mu_{r}=1$ and $\mu_{s}=10$.

Figure 2 shows the average minimum total transmit energies $\mathrm{E}\left[\mathcal{E}_{\mathrm{mrc}}^{*}\right]$ and $\mathrm{E}\left[\mathcal{E}_{\text {egc }}^{*}\right]$ needed to satisfy the outage probability target $p$ for the optimum energy allocation strategies developed in Section IV. Direct transmission results (where $\mathcal{E}_{s}^{*}=\frac{\rho}{G_{s}}$ ) are also included for comparison. These results show that the average total transmit energy decreases for both MRC and EGC as the relay channel becomes more advantaged and as $p \rightarrow 1$. As expected, MRC is more energy efficient than EGC. The energy gap between MRC and EGC grows as the relay channel becomes less advantaged, implying that knowledge of the channel state at the destination is more critical when the relay does not have a clearly advantaged channel. Cooperative transmission with MRC or EGC achieves a fixed outage probability with less average total energy than direct transmission in most cases, especially for $p \rightarrow$ 0 . Direct transmission outperforms cooperative transmission with EGC, however, when the relay's channel is statistically symmetric or disadvantaged and $p \rightarrow 1$.

Figure 3 shows the energy ratio $10 \log _{10} \frac{\mathrm{E}\left[\mathcal{E}_{s}^{*}\right]}{\mathrm{E}\left[\mathcal{E}_{r}^{*}\right]}$ as a function of $p$. These results show that the energy ratio tends to be smaller for EGC than MRC, implying that the relay assumes a larger role in cooperative communication systems where the destination does not have reliable CSI and uses EGC. As $p \rightarrow 1$, the relay tends to transmit with less relative energy for both MRC and EGC. For $p \rightarrow 0$, the relay tends to take on a larger fraction of the average total energy burden. The exception to both of these trends occurs when the relay has an advantaged channel to the destination. In this case, the results in Figure 2 show that both the source and the relay benefit from the advantaged relay channel. The relay, however, tends to experience a greater reduction in optimum transmit energy than the source due to its advantaged channel to the destination.

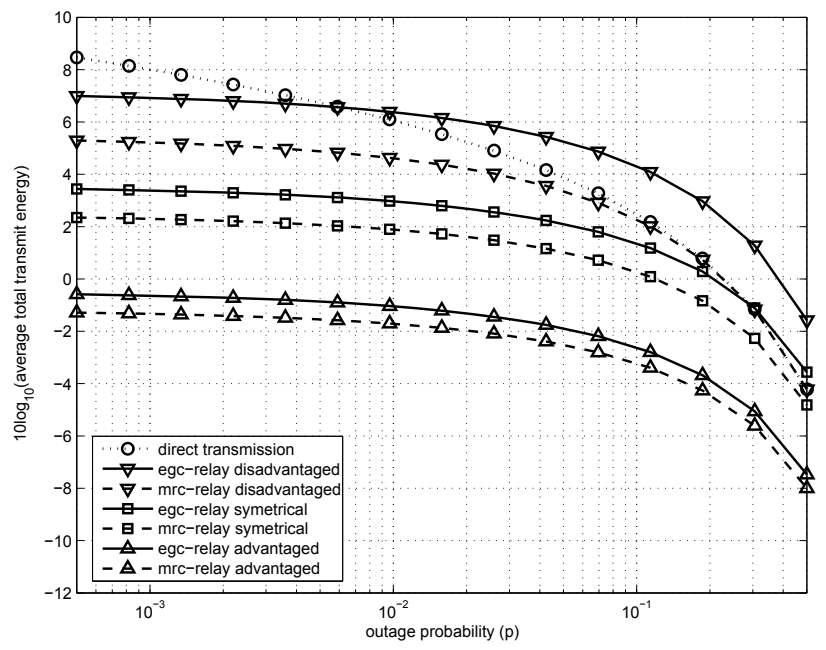

Fig. 2. Average minimum total transmission energy vs. outage probability $p$. Direct transmission results are included for comparison.

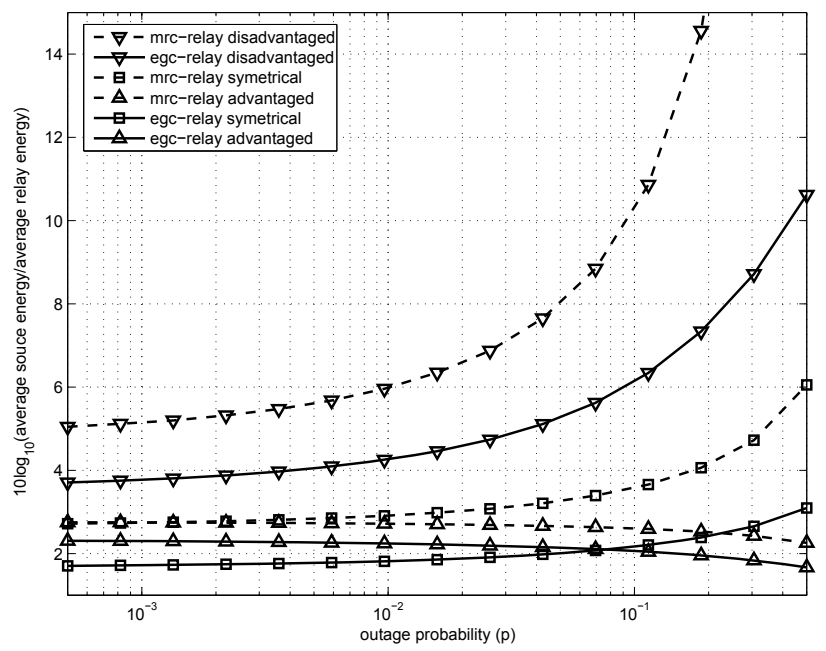

Fig. 3. Optimum average source to relay energy allocation ratio vs. outage probability $p$.

\section{CONCLUSIONS}

This paper examines the impact of receiver diversity combining on optimum energy allocation and energy efficiency of a wireless communication system with one source, one relay, and one destination using the amplify-and-forward protocol. The source is required to satisfy an outage probability constraint and both the source and relay are assumed to transmit with knowledge of the channel amplitudes. We derive the optimum energy allocation strategy for 
EGC and compare the energy efficiency and allocation to the MRC results derived in [7]. We show that, unlike MRC, optimum cooperative transmission with EGC always requires transmission by the relaying node. Numerical results for independent Rayleigh fading channels show that the ratio of optimum relay to source transmission energy tends to be greater for EGC than MRC. Our results also show that, while cooperative transmission with MRC is always the most efficient strategy, cooperative transmission with EGC tends to be more energy efficient than direct transmission when the outage probability constraint is small or when the relay experiences an advantaged channel to the destination.

\section{APPENDiX I: Proof OF Proposition 1}

To prove $\mathcal{E}_{\text {egc }}$ is convex, and hence has a unique minimum on $\mathcal{A}$, we will show that

$$
\frac{\partial^{2} \mathcal{E}_{\text {egc }}}{\partial \mathcal{E}_{s}^{2}}=\frac{H \rho \mathrm{f}(y)}{2 G_{r}\left(H \mathcal{E}_{s}-\rho\right)^{4}\left(G_{s} H \rho\left(2 H \mathcal{E}_{s}+G_{s} \mathcal{E}_{s}-2 \rho\right)\right)^{\frac{3}{2}}} \geq 0 .
$$

The function

$$
\mathrm{f}(y)=\frac{\left(y-\rho G_{s}\right)^{4} \mathrm{r}(y)}{\left(2 H+G_{s}\right)^{2} \rho^{3} G_{s}^{2}},
$$

where $y:=\sqrt{G_{s} H \rho\left(2 H \mathcal{E}_{s}+G_{s} \mathcal{E}_{s}-2 \rho\right)}$. Note that $H \rho \geq 0$ and the denominator of (6) is nonnegative on $\mathcal{A}$. Hence, the condition $\frac{\partial^{2} \mathcal{E}_{\text {egc }}}{\partial \mathcal{E}_{s}^{2}} \geq 0$ on $\mathcal{A} \Leftrightarrow \mathrm{f}(\mathrm{y}) \geq 0$ on $\mathcal{C}$, where $\mathcal{C}=[0,2 \rho H]$. The function $r(y)$ can be written as

$$
\begin{aligned}
\mathrm{r}(y)= & y^{4}+4 G_{s} \rho y^{3}-\left(12 \rho^{2} G_{s} H+3 \rho G_{s}^{2}+6 \rho G_{s} H\right) y^{2} \\
& +\left(16 \rho^{3} H^{2} G_{s}+8 \rho^{2} H G_{s}^{2}+16 \rho^{2} H^{2} G s\right) y \\
& +4 G_{s}^{2} H^{2} \rho^{4}+2 G_{s}^{3} H \rho^{3}+4 H^{2} G_{s}^{2} \rho^{3} .
\end{aligned}
$$

Note that $\frac{\left(y-\rho G_{s}\right)^{4}}{\left(2 H+G_{s}\right)^{2} \rho^{3} G_{s}^{2}} \geq 0$. Hence, the condition $\frac{\partial^{2} \mathcal{E}_{\text {egc }}}{\partial \mathcal{E}_{s}^{2}} \geq 0$ on $\mathcal{A} \Leftrightarrow \mathrm{r}(y) \geq 0$ on $\mathcal{C}$. We consider the behavior of $\mathrm{r}(y)$ in two separate cases: $G_{s} \leq 4 \rho H$ and $G_{s}>4 \rho H$.

Claim 1: $r(y) \geq 0$ on $\mathcal{C}$ when $G_{s} \leq 4 \rho H$.

Proof: Observe that $\mathrm{r}(0)=4 G_{s}^{2} H^{2} \rho^{4}+2 G_{s}^{3} H \rho^{3}+$ $4 H^{2} G_{s}^{2} \rho^{3} \geq 0$. To prove that $r(y) \geq 0$ on $\mathcal{C}$, it is only necessary to prove that $r(y)$ is non-decreasing on $\mathcal{C}$. This will be shown by proving that the minimum of $\mathrm{s}(y):=\frac{\partial \mathrm{r}(y)}{\partial y}$ is nonnegative. We can write

$$
\begin{array}{r}
\mathrm{s}(y)=4 y^{3}+12 G_{s} \rho y^{2}-2\left(12 \rho^{2} G_{s} H+3 \rho G_{s}^{2}+6 \rho G_{s} H\right) y \\
+16 \rho^{3} H^{2} G_{s}+8 \rho^{2} H G_{s}^{2}+16 \rho^{2} H^{2} G s .
\end{array}
$$

It can be shown that $\frac{\partial^{2} \mathrm{~s}(y)}{\partial y^{2}} \geq 0$ on $\mathcal{C}$. Hence, $\mathbf{s}(y)$ is convex and has a unique minimum on $\mathcal{C}$. The function $\mathrm{s}(y)$ achieves its unique minimum at the point $y^{*}:=\arg \min _{y \in C} \mathrm{~s}(y)$. This point can be written as

$$
y^{*}=-G_{s} \rho+\sqrt{4 G_{s}^{2} \rho^{2}+8 \rho^{2} G_{s} H+4 G_{s} \rho H+2 G_{s}^{2} \rho} .
$$

It can be shown that $\mathrm{s}\left(y^{*}\right) \geq 0$ when $G_{s} \leq 4 \rho H$. Hence, $\frac{\partial \mathrm{r}(y)}{\partial y} \geq 0$ for $y \in \mathcal{C}$ and $r(y)$ is non-decreasing on $\mathcal{C}$. Since $r(0) \geq 0$, this result implies that $\mathcal{E}_{\text {egc }}$ is convex on $\mathcal{A}$ when $G_{s} \leq 4 \rho H$.

Claim 2: $r(y) \geq 0$ on $\mathcal{C}$ when $G_{s}>4 \rho H$.

Proof: Observe that $r(0) \geq 0$ and $r(2 \rho H) \geq 0$. It is suffient to show that $r(y)$ is concave on $\mathcal{C}$ to imply that $r(y) \geq 0$. It can be shown that $\mathrm{u}(y):=\frac{\partial^{2} \mathrm{r}(y)}{\partial y^{2}}$ is convex. It can also be shown that $\mathrm{u}(0) \leq 0$ and $\mathrm{u}(2 \rho H) \leq 0$ when $G_{s}>4 \rho H$. Hence, $\mathrm{u}(y) \leq 0$ on $\mathcal{C}$. This implies that $\mathrm{r}(\bar{y})$ is concave, which implies that $\mathrm{r}(\bar{y}) \geq 0$ on $\mathcal{C}$ when $G_{s}>4 \rho H$.
Claim 1 and Claim 2 imply that $\mathcal{E}_{\text {egc }}$ is convex on $\mathcal{C}$.

\section{APPENDIX II: Proof OF PROPOSITION 2}

Given the convexity of $\mathcal{E}_{\text {egc }}$ on $\mathcal{A}$, we can determine whether the unique minimum of $\mathcal{E}_{\text {egc }}$ on $\mathcal{A}$ occurs at the point $\mathcal{E}_{s}=\frac{2 \rho}{G_{s}}$ by evaluating $\frac{\partial \mathcal{E}_{\text {egc }}}{\partial \mathcal{E}_{s}}$ at this point. If $\frac{\partial \mathcal{E}_{\text {egc }}}{\partial \mathcal{E}_{s}}>0$ at $\mathcal{E}_{s}=\frac{2 \rho}{G_{s}}$, then the minimum of $\mathcal{E}_{\text {egc }}$ on $\mathcal{A}$ must occur at $\mathcal{E}_{s}<\frac{2 \rho}{G_{s}}$ (corresponding to $\mathcal{E}_{r}^{*}>0$ ), otherwise the minimum occurs at $\mathcal{E}_{s}=\frac{2 \rho}{G_{s}}$ (corresponding to $\mathcal{E}_{r}^{*}=0$ ). It can be shown that

$$
\left.\frac{\partial \mathcal{E}_{\mathrm{egc}}}{\partial \mathcal{E}_{s}}\right|_{\mathcal{E}_{s}=\frac{2 \rho}{G_{s}}}=1>0
$$

hence the unique minimum of $\mathcal{E}_{\text {egc }}$ on $\mathcal{A}$ must occur at $\mathcal{E}_{s}<\frac{2 \rho}{G_{s}}$. This implies that $\mathcal{E}_{r}^{*}>0$ for all $G_{s}, G_{r}, H, \rho$.

\section{REFERENCES}

[1] A. Sendonaris, E. Erkip, and B. Aazhang, "Increasing uplink capacity via user cooperation diversity," in IEEE International Symp. on Information Theory, August 16-21 1998, p. 156.

[2] A. Sendonaris, E. Erkip, and B. Aazhang, "User cooperation diversity — part i: System description," IEEE Transactions on Communications, vol. 51, no. 11, pp. 1927-1938, Nov. 2003.

[3] A. Sendonaris, E. Erkip, and B. Aazhang, "User cooperation diversity — part ii: Implementation aspects and performance analysis," IEEE Transactions on Communications, vol. 51, no. 11, pp. 1939-1948, Nov. 2003.

[4] J. N. Laneman, D. N. C. Tse, and G. W. Wornell, "Cooperative diversity in wireless networks: Efficient protocols and outage behavior," IEEE Transactions on Information Theory, vol. 50, no. 12, pp. 3062-3080, 2004.

[5] I. Maric and R. D. Yates, "Bandwidth and power allocation for cooperative strategies in gaussian relay networks," in Proceedings of the Asilomar Conference on Signals, Systems, and Computers, 2004, vol. 2, pp. 1907-1911 Vol.2.

[6] D. R. Brown III, "Resource allocation for cooperative transmission in wireless networks with orthogonal users," in Conference Record of the Thirty-Eighth Asilomar Conference on Signals, Systems and Computers, , 2004, vol. 2, pp. 1473 1477 Vol.2.

[7] Jie Yang and D.R. Brown III, "The effect of channel state information on optimum energy allocation and energy efficiency of cooperative wireless transmission systems," Conference on Info. Sciences and Systems (CISS), pp. 1044-1049, 2006.

[8] A. Host-Madsen and Junshan Zhang, "Capacity bounds and power allocation for wireless relay channels," IEEE Trans. on Information Theory, vol. 51, no. 6, pp. 2020-2040, 2005.

[9] D. Gunduz and E. Erkip, "Outage minimization by opportunistic cooperation," in International Conference on Wireless Networks, Communications and Mobile Computing, 2005, vol. 2, pp. 1436-1442.

[10] J. Luo, R. S. Blum, L. J. Cimini, L. J. Greenstein, and A. M. Haimovich, "Decode-and-forward cooperative diversity with power allocation in wireless networks," in IEEE Global Telecommunications Conference, 2005, vol. 5, p. 5.

[11] J. N. Laneman, G. W. Wornell, and D. N. C. Tse, "An efficient protocol for realizing cooperative diversity in wireless networks," in Proceedings of the IEEE International Symposium on Information Theory (ISIT), June 24-29 2001, p. 294. 